pneumococcal vaccinations have been recommended prior to initiating treatment with biological agents. The COMEDRA trial, conducted in France, revealed the usefulness of a nurse-led consultation in the management of RA-associated comorbidities. Despite this, however, only $40 \%$ of patients were shown to be appropriately vaccinated (1).

Objectives: This study sought to better understand the obstacles encountered in RA patients as to influenza and pneumococcal vaccinations, along with their motivations.

Methods: This qualitative study was conducted at the rheumatology department by means of semi-directed nurse-led interviews lasting about 45 minutes, between July and November 2015. Four topics were analyzed: obstacles and motivations to influenza vaccination, obstacles and motivations to pneumococcal vaccination, post-vaccination follow-up, and information sources. The interviews were registered and transcribed within 45 hours post-interview, and were pursued until data saturation. Transversal data analysis was carried out by the nurse in charge of the interview, then by a second nurse, with the two analyses assembled according to the traditional concept of double reading. Differences between both analyses were thoroughly discussed, with a consensus sought by a third researcher, namely a rheumatologist, as necessary.

Results: Overall, 15 interviews were conducted involving 11 women and 4 men, with a mean age of 63 years (29-83). All patients were suffering from RA and undergoing at least one immunosuppressant therapy. Most $(80 \%)$ were vaccinated against pneumococcus, but only $33 \%$ against influenza, with eight patients declaring having been affected by influenza and one by pneumococcusrelated pulmonary disease.

The obstacles to vaccinations, revealed during the interviews, primarily concerned fears of unwanted effects in relation with the vaccinations, particularly concerning the influenza vaccination. The patients also reported anxieties in relation with the vaccines' excipients, with overdoses, or with disease reactivation. Moreover, media impact, contradictory information, the fact that vaccinations were not always proposed, and the lack of traceability may also be considered obstacles against vaccinations. Influenza is often considered a benign disease, and the vaccine poorly efficacious. Patients with prior influenza were more motivated towards vaccination. The primary motivation reported by the patients, and especially as to the pneumococcal vaccination, was the protection provided by the vaccine. The perception of frailty and increased infection risk associated with the disease and its treatments were other sources of motivation, and particularly when vaccination was highly recommended by the physicians, and when there had been no public controversy.

Conclusions: The fears and motivations associated with these two vaccinations are not identical. Influenza vaccination appears to be poorer perceived, patients reporting more fears related to it. With respect to pneumococcus vaccination, we only collected a few comments, given that the vaccine is less well know, and thus better accepted.

References:

[1] Gossec L. Arthritis Rheumatol 2016; 68 (suppl 10).

Disclosure of Interest: None declared

DOI: 10.1136/annrheumdis-2017-eular.4994

\section{SAT0734-HPR THE EFFECT OF RHEUMATIC DISEASES ON WORK ABILITY: A STUDY OF SELF-ASSESSMENT OF SF-36 IN PATIENTS WITH SMART SYSTEM OF DISEASE MANAGEMENT (SSDM)}

H. Song ${ }^{1}$, J. Gu ${ }^{2}$, H. Wu ${ }^{3}$, S. Li ${ }^{4}$, Y. Zhao ${ }^{5}$, H. Sun ${ }^{6}$, J. Huang ${ }^{7}$, X. Li ${ }^{8}$, R. Wu ${ }^{9}$, B. Wu ${ }^{10}$, C. Xiao $^{11}$, L. Qian ${ }^{12}$, F. Xiao ${ }^{13}$, H. Xiao ${ }^{13}$, M. Wang ${ }^{13}$ Y. Jia ${ }^{13}$, Z. Zhang ${ }^{5} .{ }^{1}$ Department of Rheumatology and Immunology, Department of Rheumatology and Immunology, Beijing; ${ }^{2}$ The Third Affiliated Hospital of Sun Yat-sen University, Guangzhou: ${ }^{3}$ Department of Rheumatology and Immunology, Dongguan People's Hospital, Dongguan; ${ }^{4}$ The First Affiliated Hospital of PLA General Hospital, Beijing; ${ }^{5}$ Department of Rheumatology and Immunology, The First Affiliated Hospital of Harbin Medical University, Harbin ${ }^{6}$ Shandong Provincial Hospital, Jinan; ${ }^{7}$ The sixth Affiliated Hospital of Sun Yat-sen University, Guangzhou: ${ }^{8}$ Anhui Medical University Affiliated Provincial Hospital, Hefei; ${ }^{9}$ The First Affiliated Hospital of Nanchang University, Nanchang, ${ }^{10}$ The First People's Hospital of Jingzhou, Jingzhou; ${ }^{11}$ TCM-Integrated Hospital of Southern Medical University, Guangzhou; ${ }^{12}$ The Second Affiliated Hospital of Medical University of Anhui, Hefei; ${ }^{13}$ Medical Department, Shanghai Gothic Internet Technology Co., Ltd., Shanghai, China

Background: A variety of rheumatic diseases can significantly affect the patient's quality of life and work ability. SF-36 is a commonly used tool to assess the quality of life and work ability in patients with chronic disease. In the past, most patients were guided by doctors/nurses to assess SF-36 in a paper form. At present, there is a lack of research on the quality of life in patients with rheumatic diseases by using new mobile tools in the real world.

Objectives: To explore the effect to the life ability of rheumatic disease and the potential association between the disease activity and SF-36 in patients with rheumatoid arthritis (RA) using SSDM.

Methods: SSDM is a new smart disease management mobile tool, which includes physicians' and patients' application system. After entering the results of SF-36 assessment by patients, all data can be synchronized automatically to the mobile terminal of authorized rheumatologist. According to the scores of SF-36 $(<12.5$,
$12.5-50$ and $>50$ ), the quality of life was divided into three levels: poor, moderate and good.

Results: From June 2016 January 2017, data were extracted online from the mobile terminals of 839 adult patients (284 male and 555 female) in 62 hospitals across China. All patients performed self-assessment of SF-36 for a total of 1,065 times. The mean age was $38.12 \pm 13.87$ (18 to 81 ) years and the median disease duration was 20.60 ( 0 to 573 ) months. There are 25 kinds of rheumatic diseases involved, including RA (23\%), ankylosing spondylitis (AS, 19\%), systemic lupus erythematosus (SLE, 13\%), osteoarthritis (OA, 11\%), Sjogren syndrome (SS, 10\%), polymyositis/dermatomyositis (PM/DM, 6\%), mixed connective tissue disease (MCTD, $6 \%$ ) and others (12\%).

The 8 items of SF-36 index were averaged between 49.07 (General Health perceptions, GH) to 74.12 (Physical Functioning, PF). The overall mean score of patients with different rheumatic diseases was higher than 50 . However, there were 5 kinds of rheumatic diseases with average score of 5 items less than 50 , including: MCTD, SS, PM/DM, AS and SLE in patients with GH score: 44.24, 44.60, 47.87, 47.93 and 49.16, PM/DM in patients with the Emotional Role functioning (RE): 47.52, Physical Role functioning (RP): 44.68 and AS patients with Reported Health Transition (HT): 49.64, respectively. In RA patients, $38 \%$ and $37 \%$ of patients reported that their ability to work were affected by disease and mood changes respectively. Bivariate correlation analysis showed that DAS28 was negatively correlated with the two item scores (RP and RE) of SF-36 index in RA patients, $p<0.01$.

Conclusions: SSDM can be used to assess SF-36 in patients with rheumatic disease. The Quality of life scores in patients with MCTD, SS, PM/DM, AS, and SLE were more likely to be affected by rheumatic disease. The disease activity (DAS28) of RA patients was negatively correlated with the work ability (RP and RE of SF-36 score)

Disclosure of Interest: None declared

DOI: 10.1136/annrheumdis-2017-eular.4990

\section{SAT0735-HPR PATIENTS' ASSESSMENT OF THE APPLICABILITY OF THE ULTRASOUND IMAGE METHOD}

J.A. Mendonça ${ }^{1}$, W.H. Rodrigues Ferreira ${ }^{2}$, I.M.M. Laurindo ${ }^{3} .{ }^{1}$ Rheumatology, Pontifical Catholic University of Campinas, Campinas, ${ }^{2}$ Rheumatology, Grupari Petrópolis, Petrópolis; ${ }^{3}$ Rheumatology, Universidade Nove de Julho, São Paulo, Brazil

Background: The applicability of ultrasonography in diagnosis and monitoring of rheumatic diseases has been steadily growing but little is known about the patient's perspective.

Objectives: To assess the patient's knowledge of the ultrasound (US) method and its clinical applicability as well as to evaluate how much was learned after a lecture.

Methods: Patients with rheumatic diseases were invited to an educational meeting that included an US lecture. They were asked to fill out a questionnaire before and after the 35-minute lecture about US and its clinical uses. The questionnaire included questions about their disease and treatment, their educational background (years of study) and ten questions about US and its clinical uses (table). These ten questions had to be answered with "yes", "no", or "I am not sure".

Results: A total of 70 patients attended the lecture and answered the questionnaires. Their demographic characteristics were as follows: Mean \pm SD: age $63 \pm 12$ years, disease duration: $9.6 \pm 0.8$ years, the most frequent self- reported diagnosis was rheumatoid arthritis $(57,2 \%)$, while osteoarthritis accounted for another $20 \%$. Most patients had only eight years of study $(64.3 \%)$ and a university degree was reported by $14 \%$. The questionnaire responses of 66 patients with rheumatic diseases were analysed. There was a noticeable increase in the positive responses to these questions: 1, 2, 3 and 7 , after the lecture, $44 \%$

Table - Questions :
1)Do you know what arthritis is?
2)Do you know what an ultrasound exam is?
3) Do you believe that an ultrasound exam could help in the diagnosis of your disease?
4) Do you believe that an ultrasound exam could help in monitoring your treatment?
5)Have you ever had one of your joints examined by ultrasound?
6) Has the exam changed your treatment?
happening to your joints?
8)Do you think that an ultrasound exam would help you follow the treatment?
9) Do you believe that an ultrasound exam can detect inflammation in your joints even when
it is not clinically diagnosable by your doctor?

10)Do you think that an ultrasound exam would help you to understand your illness better? 
$(p=0.001) ; 29 \%(p=0.001) ; 10 \%(p=0.06) ; 27 \%(p=0.009)$, respectively. Although only $26 \%$ of the patients have had an articular US, in $65 \%$ it led to a change in treatment. Positive answers to some questions, which were perhaps too complex, showed a discrete increase, less than $10 \%$, conceivably due to the surprisingly high background of positive answers.

Conclusions: As expected, the US image method was familiar to most but not its applicability in articular diseases. After the lecture there was an increase in understanding of the positive impact of US in rheumatic diseases, implying that short and focused lectures are a useful tool in educational programs for patients.

Disclosure of Interest: None declared

DOI: 10.1136/annrheumdis-2017-eular.1426

\section{SAT0736-HPR "LIKE A BAG OF LIQUORICE ALLSORTS - EVERYBODY'S GOT DIFFERENT FLAVOURS": A QUALITATIVE FOCUS GROUP STUDY TO EXPLORE SYMPTOMS OF FATIGUE, SLEEP DISTURBANCES AND PAIN IN PRIMARY SJÖGREN'S SYNDROME PATIENTS AND TO DEVELOP A FUTURE MODEL OF CARE}

K.L. Hackett ${ }^{1}, 2$, V. Deary ${ }^{2,3}$, K. Deane ${ }^{4}$, J.L. Newton ${ }^{1,2}$, W.-F. Ng $^{1,2}$, T. Rapley ${ }^{1}$ ${ }^{1}$ Newcastle University; ${ }^{2}$ Newcastle upon Tyne NHS Foundation Trust; ${ }^{3}$ Northumbria University, Newcastle upon Tyne; ${ }^{4}$ University of East Anglia, Norwich, United Kingdom

Background: Fatigue, sleep disturbances and pain, are symptoms of primary Sjögren's syndrome (PSS). However, current clinical interventions predominantly focus on treating patients' dryness symptoms.

Objectives: To explore the experience of fatigue, sleep disturbances and discomfort in people with PSS, to investigate the impact of these symptoms on patients' daily lives and to develop an intervention strategy to address them.

Methods: Qualitative focus groups with open-ended questions allowed participants to explore ideas together and focus on issues they perceived as being important. PSS patients $(n=10)$ and spouses $(n=3)$ took part in three focus groups divided into six sessions which were facilitated by two clinician researchers using a topic guide. Discussion topics included; the symptoms, strategies used by patients used to manage their symptoms, possible future intervention components proposed from the literature and possible modes of delivering future interventions. The meetings were audio-recorded, transcribed verbatim and analysed using thematic analysis ${ }^{1}$.

Results: Patient participants all experienced these symptoms. Symptom severity varied within individuals and flares occurred unpredictably. Fatigue, sleep disturbances and discomfort, all affected patients' lives and those around them and sometimes felt overwhelming. Discomfort symptoms included oral and ocular dryness, tingling, nausea and difficulties tolerating light and noise. The invisible nature of these features meant patients often struggled to meet others' expectations, which affected their mood and resulted in social withdrawal. Fatigue was a major barrier to engaging in work, productivity and leisure activities. Sleep disturbances further compounded the fatigue. Patients employed a range of strategies to self-manage their symptoms to varying degrees of success.

Participants expressed a need for tailored support from health care professionals which included information provision, access to peer support and professional support to apply symptom management information. A three stepped model of care was proposed. The model includes different modes of delivering intervention content, including written information, education groups, peer support, digital self-management and one-to-one therapy. Intervention intensity increased with each step in the model.

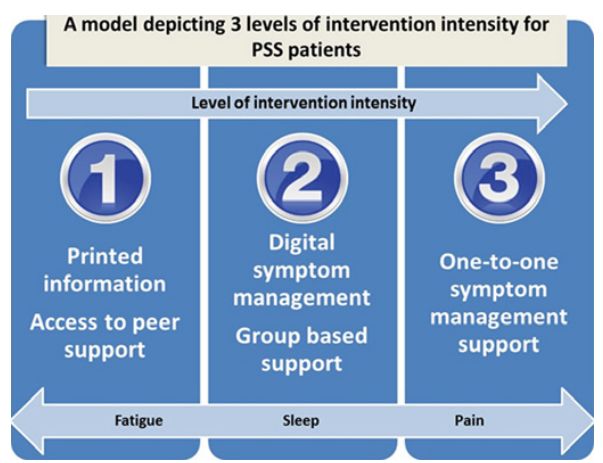

Conclusions: Symptoms of fatigue, sleep disturbances and discomfort all impact on PSS patients' daily lives and individualised interventions are needed to support self-management. Care needs to be tailored as different patients require variable levels of support. A stepped model of future symptom management delivery is proposed.

References:

[1] Braun V and Clarke V (2006) Using thematic analysis in psychology. Qualitative

Research in Psychology, 3(4), 77-101.

Acknowledgements: This project was funded by Arthritis Research UK (grant 20169) and the United Kingdom Occupational Therapy Research Foundation.
Disclosure of Interest: None declared

DOI: 10.1136/annrheumdis-2017-eular.2823

\section{SAT0737-HPR FACILITATORS AND BARRIERS FOR PARTICIPATION IN PHYSICAL ACTIVITIES IN JUVENILE IDIOPATHIC ARTHRITIS PATIENTS AND HEALTHY CONTROLS}

K. Risum ${ }^{1}$, A.M. Selvaag ${ }^{1}$, Ø. Molberg ${ }^{1}$, H. Dagfinrud ${ }^{2}$, H. Sanner ${ }^{1} .{ }^{1}$ Os/o University Hospital; ${ }^{2}$ Diakonhjemmet Hospital, Oslo, Norway

Background: Knowledge is sparse regarding facilitators and barriers for participation in physical activity (PA) in patients with juvenile idiopathic arthritis (JIA) and whether they differ from controls. Furthermore, knowledge about preferences for leisure time physical activities and participation in physical education (PE) in school in JIA patients is limited.

Objectives: To explore participation in PA and PE in JIA patients, and to explore facilitators and barriers for PA participation in JIA and matched controls.

Methods: The study cohort included $60 \mathrm{JIA}$ patients (50 girls, 10 boys) and 60 controls individually matched for age and sex randomly selected from the Norwegian Population Registry. Of the JIA patients, 30 had persistent oligoarthritis and 30 had polyarticular disease (extended oligoarthritis and polyarticular RF $+/$-). The patients were aged 10-16 years and recruited consecutively at Oslo University Hospital in 2015. Participation in PA and facilitators and barriers for PA participation, were explored with structured interviews. The interview guide was developed for this study based on literature review and clinical experience. Differences between the study groups were analyzed using the McNemar test.

Results: Participation in physical activities was not significantly different between JIA patients and controls (Table 1). The most commonly practiced organized physical activities in both groups were dancing and soccer, and the most commonly practiced unorganized physical activities were jogging, training at fitness center and strength exercising at home. Participation in PE is shown in Table 1. Fun was the most reported facilitator for participation in PA both in patients and controls, $40(67 \%)$ vs $45(75 \%)$, respectively, $\mathrm{p}=0.32$. Becoming fit was an often reported facilitator in patients and controls, $12(20 \%)$ vs $21(35 \%)$ respectively, $p=0.07$. Being with friends was a facilitator more often reported by controls than patients, $13(22 \%)$ in controls vs $1(2 \%)$ patient $(p<0.001)$. Less pain was a facilitator in 4 patients, but not in any controls $(p=0.06) .26(43 \%)$ patients and $19(32 \%)$ controls reported barriers for participating in PA $(p=0.46)$. More controls reported time as a PA barrier, $11(18 \%)$ vs $3(5 \%)(p=0.02)$, while more patients reported pain as a barrier; $18(30 \%)$ vs $8(13 \%)(p=0.03)$. Disease activity was a barrier in 4 (7\%) patients.

Table 1. Participation in physical activity (PA) and physical education (PE)

\begin{tabular}{lccc}
\hline & JIA $(n=60)$ & Controls $(\mathrm{n}=60)$ & p-value \\
\hline Participation in PA (organized/unorganized) & $51(85)$ & $56(93)$ & 0.14 \\
Participation in organized PA & $38(63)$ & $47(78)$ & 0.11 \\
Participation in unorganized PA & $41(68)$ & $42(70)$ & 1.00 \\
Participation in PE & $42(70)$ & $59(98)$ & $<0.001$ \\
Always & $16(27)$ & $0(0)$ & \\
Always (sometimes with modification) & $2(3)$ & $1(2)$ & \\
Sometimes & &
\end{tabular}

Numbers are $\mathrm{n}(\%)$

Conclusions: The majority of JIA patients and controls participated in organized or unorganized PA. Fun was the most reported facilitator in patients, followed by becoming fit and having less pain. Pain was the dominant PA barrier in patients, while time was the most frequently reported barrier in controls. Some patients experienced disease activity as a barrier. Nearly all the patients (97\%) participated regularly in $\mathrm{PE}$, but $1 / 4$ needed some modifications. These results are reassuring, and highlight the importance of enjoyment for participation in PA Disclosure of Interest: None declared

DOI: 10.1136/annrheumdis-2017-eular.6063

\section{SAT0738-HPR EXPERIENCES OF TELE-HEALTH FOLLOW-UP IN PATIENTS WITH RHEUMATOID ARTHRITIS: A QUALITATIVE INTERVIEW STUDY}

L.R. Knudsen ${ }^{1}$, A. de Thurah ${ }^{1,2}$, K. Lomborg ${ }^{2,3}$. ${ }^{1}$ Department of Rheumatology, Aarhus University Hospital; ${ }^{2}$ Department of Clinical Medicine, Aarhus University ${ }^{3}$ The Research Programme in Patient Involvement, Aarhus University Hospital, Aarhus, Denmark

Background: Recently, the effectiveness of monitoring disease activity in rheumatoid arthritis (RA) through patient reported outcome (PRO) based telehealth follow-up was tested in a randomized controlled trail, TeRA (1). The TeRA study evaluated the effectiveness of the tele-health follow-up but did not study how patients grasp this new form of disease control. Many studies on tele-medicine focus on the over-all patient satisfaction with tele-medicine follow-up, but gives limited insight into what drives patient satisfaction (2).

Objectives: To explore the experiences of a PRO based tele-health follow-up from the perspective of patients with RA with a special attention on experiences of taking a more active role and assuming more responsibility in the disease control. Methods: The qualitative research strategy was Interpretive Description (3). From October 2015 to January 2016 we conducted individual semi-structured interviews 